\title{
Effects of PTH treatment on tibial bone of ovariectomized rats assessed by in vivo micro-CT
}

\author{
J. E. M. Brouwers • B. van Rietbergen • R. Huiskes • \\ K. Ito
}

Received: 23 September 2008 / Accepted: 15 December 2008 /Published online: 5 March 2009

(C) The Author(s) 2009. This article is published with open access at Springerlink.com

\begin{abstract}
Summary Using in vivo microcomputed tomography (micro$\mathrm{CT}$ ), we found in parathyroid hormone (PTH)-treated osteopenic rats linear increases in cortical and trabecular, due to increased trabecular thickness and number, bone mass. Bone was formed in cavities, leading to restoral of nearly cleaved trabeculae. For the first time, effects in PTH-treated rats were analyzed longitudinally.

Introduction Our aims were to over time (1) determine changes in trabecular thickness and number after PTH, (2) compare responses to PTH between the meta- and epiphysis, (3) determine effects of PTH on mineralization and mechanical properties, (4) determine locations of new bone formation due to PTH on a microlevel, and (5) determine the predictive value of bone structural properties for gain in bone mass after PTH.

Methods Adult rats were divided into ovariectomy (OVX; $n=8)$, SHAM-OVX $(n=8)$, and OVX and PTH treatment $(n=9)$. Between weeks 8 and 14 , PTH rats received daily subcutaneous PTH injections $(60 \mu \mathrm{g} / \mathrm{kg} /$ day $)$.
\end{abstract}

J. E. M. Brouwers · B. van Rietbergen · R. Huiskes · K. Ito Department of Biomedical Engineering,

Eindhoven University of Technology,

Eindhoven, The Netherlands

J. E. M. Brouwers

e-mail: j.e.m.brouwers@tue.nl

R. Huiskes

e-mail: h.w.j.huiskes@tue.nl

K. Ito

e-mail: k.ito@tue.nl

B. van Rietbergen $(\bowtie)$

Technische Universiteit Eindhoven,

P.O. Box 513, 5600 MB Eindhoven, The Netherlands

e-mail: b.v.rietbergen@tue.nl
At weeks $0,8,10,12$, and 14, in vivo micro-CT scans were made of the proximal and diaphyseal tibia. After sacrifice, all tibiae were tested in three-point bending.

Results PTH increased bone volume fraction linearly over time in meta- and epiphysis, accompanied by increased trabecular thickness in both and increased trabecular number only in the latter one. CT-estimated mineralization increased in trabecular and remained constant in cortical bone. Ultimate load and energy were increased and ultimate displacement and stiffness unaltered compared to SHAM rats. For those trabeculae analyzed, bone was formed initially on places where it was most beneficial for increasing their strength and later on to all surfaces.

Keywords Bone microstructure · In vivo micro-CT . Osteoporosis $\cdot$ PTH $\cdot$ Rat ovariectomy model

\section{Introduction}

Daily injections of parathyroid hormone (PTH) have anabolic effects on bone and are Food and Drug Administration approved for treatment of vertebral fractures associated with postmenopausal osteoporosis. The effects of PTH have been extensively studied in the ovariectomized rat. This is an animal model that has been shown to be a good first predictor of treatment potential of a drug for osteoporosis and as such is commonly used. PTH markedly increases trabecular bone mass in the proximal tibia, femoral neck, and lumbar vertebra of ovariectomized, aged, and young rats [1-15]. Additionally, it increases cortical width, cortical bone area, and axial moments of inertia as a result of mostly endocortical bone formation, leading to reduced bone marrow cavities and, to a lesser extent, increased periosteal bone formation [7, 16-18]. 
Mechanical strength in anatomical sites like the vertebra, femoral neck, and femoral diaphysis increases accordingly in rats after PTH treatment $[2-4,9]$. Although the effects of PTH have been extensively studied, some aspects are still unclear and need further research.

Although most increases in trabecular bone mass after PTH treatment have been reported to result from increased trabecular thickness, in a few studies in dogs, rodents, and monkeys, an increase in trabecular number was reported after PTH treatment [19-25], which is an uncommon feature in itself. The suggested mechanism for this was the observation of longitudinal tunneling of thickened trabeculae seen in histological sections as a remodeling mechanism to maintain trabecular thickness within limits. Tunneling of thickened individual trabeculae would convert them into multiple trabeculae, resulting in a normalization of trabecular thickness and an increase in trabecular number. It has been suggested that trabecular thickness will increase until it reaches a maximum, after which intratrabecular resorption will take place [23]. This suggests that changes in trabecular number and thickness may depend on the structure at the start of the treatment and may vary over time depending on dose and duration of treatment and anatomical site. It is known that the same increase in bone mass due to trabecular thickness or number has different mechanical implications, with the latter one having a higher increase in mechanical performance [26, 27]. Therefore, it is important to evaluate the changes in both trabecular thickness and number after PTH treatment over time to provide more insight into the potential of increasing mechanical performance.

In most studies on PTH in rats, the metaphyseal trabecular bone, often in the tibia, has been analyzed. It is known, however, that even in adult rats, the growth plate still shows some activity, though to a lesser extent than in young animals, which inherently influences metaphyseal trabecular bone [28]. As PTH is a naturally occurring hormone that has an essential role in the growth plate, it can be questioned whether the metaphysis would be the best predictor of the effects of PTH in postmenopausal women, in whom the growth plate has been closed since adolescence. The neighboring epiphysis, which does not undergo linear bone growth, may offer a more suitable translational site for analyzing PTH effects. Also, loading patterns have shown to be different between the meta- and epiphysis [29], with higher strains occurring in the latter one. Moreover, the response to PTH has shown to be directed toward higher strain areas in a finite element modeling study in osteoporotic patients [30] and has shown to be smaller in the caudal vertebrae, where loads are relatively low, compared to the lumbar vertebrae [31], indicating that PTH effects may be mechanically directed. Taken together, it would be highly relevant to compare the response to PTH between the meta- and epiphysis, which has not previously been done.

Conflicting results have been reported regarding the influence of PTH on the degree and heterogeneity of bone mineralization. In a study in patients, some aspects of mineralization were altered after PTH use in men and women [32]. In a study in rats, long-term treatment of rats with PTH resulted in a slightly wider variation in mineralization in the bone reflecting the newly formed bone [18]. In two other rat studies, however, no influence of PTH on mineralization was found [2, 33]. As altered mineralization due to PTH may have detrimental effects on mechanical behavior, in spite of a potentially increased bone mass, it is important to further evaluate the effects of PTH on mineralization and mechanical properties.

Most reported studies on effects of PTH in rats were cross-sectional in design and rats were mostly sacrificed after just one or two different treatment periods providing little information about how exactly microstructure and mineralization evolved over the course of treatment. Additionally, as changes in bone mass and structure could not be monitored in the same animal, no specific knowledge was obtained about how and where new bone is formed on a microlevel. Finally, it could not be determined within a subject how much bone mass had increased after PTH, which is clinically very important as the patient's response to PTH should be monitored and ideally be predicted. Recently, however, in vivo microcomputed tomography (micro-CT) scanners have become available to monitor bone microstructure in small living animals. With this tool, combined with image registration software, we were able to analyze changes in bone structure and mineralization over time after PTH treatment in ovariectomized rats and hereby address the following aims: (1) determine changes in trabecular thickness and number after PTH over time, (2) compare responses to PTH between the meta- and epiphysis, (3) determine effects of PTH on mineralization and mechanical properties, (4) determine locations of new bone formation due to PTH on a microlevel over time, and (5) determine the predictive value of bone structural properties for gain in bone mass after PTH.

\section{Materials and methods}

Animals

Twenty-five female 6-month-old virgin Wistar rats (Harlan Laboratories, Horst, The Netherlands) were allowed to acclimatize for 7 days before the start of the experiment. The rats were maintained with a cycle of $12 \mathrm{~h}$ light and $12 \mathrm{~h}$ darkness and allowed to eat and drink ad libitum. The 
experiment was approved by the Animals Ethics Committee of the University of Maastricht, The Netherlands. The rats were divided into three groups (with equal weight distributions): control $(n=8)$, ovariectomy (OVX; $n=8)$, and OVX and PTH treatment $(n=9)$. All rats were ovariectomized at week 0 and the control group underwent a SHAM ovariectomy. Success of OVX was confirmed at necropsy by determining atrophy of the uterine horns. Rats were left untreated for 8 weeks to allow for osteopenia to develop. After 8 weeks, rats in the PTH group received daily subcutaneous injections of PTH $(60 \mu \mathrm{g} / \mathrm{kg} / \mathrm{day})$ for 6 weeks. This relatively high dose was chosen to maximize the possibility of trabecular tunneling to occur and lies within the dose range investigated in a dose-dependency study [18]. Synthetic human PTH (1-34; Bachem, Bubendorf, Switzerland) was dissolved in a vehicle of acidified saline $(0.1 \mathrm{~N})$ and $2 \%$ rat serum. Body weight was measured weekly, and the PTH dose adjusted accordingly. Rats were sacrificed at 14 weeks by cervical dislocation under deep anesthesia after the final CT scan.

\section{Micro-CT scanning}

Directly after the operation, a 6-mm micro CT-scan $(70 \mathrm{kV}$, $114 \mu \mathrm{A}, 1,000$ projections per $180^{\circ}, 261 \mathrm{~ms}$ integration time) with an isotropic resolution of $15 \mu \mathrm{m}$ was made of the proximal tibia using an in vivo micro-CT scanner (vivaCT 40, Scanco Medical AG, Brütissellen, Switzerland). The CT scanner was calibrated and a beam-hardening correction algorithm was applied to all scans [34]. Another 3.15-mm micro-CT scan of the diaphysis was made with an isotropic resolution of $30 \mu \mathrm{m}(70 \mathrm{kV}, 114 \mu \mathrm{A}, 250$ projections per $180^{\circ}, 350 \mathrm{~ms}$ integration time). Before this measurement, the most distal and proximal point of the tibia was located in a scout view to ensure that the exact middle of the diaphysis was scanned. Follow-up in vivo CT scans were made after 8,10,12, and 14 weeks to monitor bone structure. Every follow-up scan was registered with the first scan by using image registration software that registers two scans based on minimizing the correlation coefficient [35]. Total scanning time was $42 \mathrm{~min}$, during which the animal was anesthetized with isoflurane and the scanned leg was placed in a custom-made leg-fixating device. The design of the rat holder was such that the left leg was not exposed to radiation while scanning the right leg. Radiation damage to the scanned bone was not expected to occur, based on a previous study, in which 8 weekly CT scans with the same radiation dose caused no detected bone damage [36]. In that study, we also showed that the reproducibility of all structural parameters was high, with a coefficient of variation of about $1 \%$.

From the CT scans, the metaphyseal trabecular bone, epiphyseal trabecular bone, metaphyseal cortical bone, and diaphyseal cortical bone were analyzed. For each analysis, the estimated mineral density of the bone tissue was determined based on the linear correlation between CT attenuation coefficient and bone mineral density (BMD).

Image processing of all scans included Gaussian filtering and segmentation as described elsewhere in detail [36]. In brief, the same filtering and segmentation values were used for every measurement of each animal (trabecular bone: sigma $=0.7$, support $=1$, threshold density $=0.575 \mathrm{~g} \mathrm{HA} / \mathrm{cc}$, equivalent to $24 \%$ of maximal grayscale value; cortical bone: sigma $=0.8$, support $=1$, threshold density $=0.642 \mathrm{~g}$ $\mathrm{HA} / \mathrm{cc}$, equivalent to $26 \%$ of maximal grayscale value). From every baseline and follow-up CT scan, the trabecular bone of the meta- and epiphyseal areas were manually selected and bone structural parameters (bone volume fraction (BV/TV), connectivity density (Conn.D), structure model index (SMI), trabecular number, thickness, and separation (Tb.N, Tb.Th, Tb.Sp)) were automatically determined (Fig. 1). Cortical bone of the metaphysis was manually selected from the hundred most distal slices. From the CT scan of the diaphysis, all slices were manually selected. Cortical thickness and polar moment of inertia (pMOI) were determined. The selected cortical bone in the meta- and diaphysis at weeks 8 and 14 was registered for all PTH-treated rats to determine to what extent bone formation over 6 weeks was due to endosteal or periosteal apposition.

\section{Trabecular tunneling}

We expected trabecular tunneling only to occur, if at all, in the thickest trabeculae; hence, for all PTH-treated rats, the meta- and epiphyseal trabecular bones of the CT scans of a)

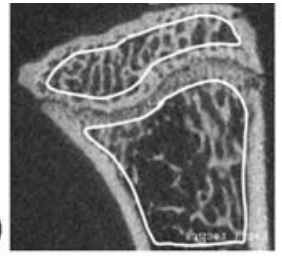

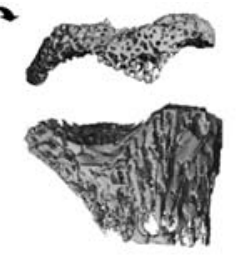

b)

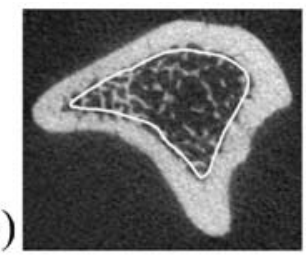

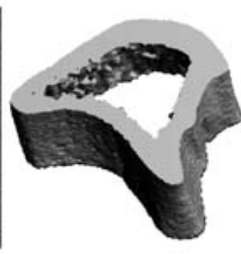

c)

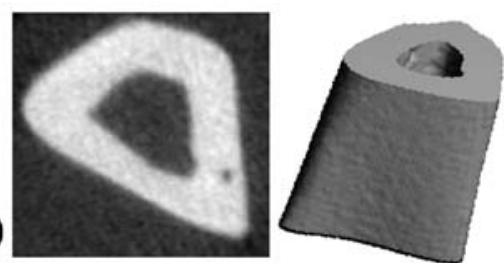

Fig. $1 \mathrm{CT}$ scan of a proximal metaphysis showing hand-drawn contours of the metaphyseal and epiphyseal trabecular bone, b proximal metaphysis showing hand-drawn contours of metaphyseal cortical bone, and $\mathbf{c}$ diaphyseal cortical bone 
weeks 12 and 14 were registered. After registration, the two CT scans were overlaid and visually checked for trabecular tunneling. This was done by going through the registered images per five slices, first from proximal to distal end and then from anterior to posterior sides while specifically looking for trabeculae that were thick at week 12 to see whether they were split in two at week 14, by using histologic sections from the literature as image to look for.

To determine whether there is a maximum trabecular thickness, after which trabecular tunneling takes place, we analyzed the distribution of trabecular thickness in the epiphysis of all rats at all time points. The scanner software provides outputs of counts per bin and trabecular thickness was categorized in bins of $15 \mu \mathrm{m}$.

Prediction of gain in bone mass after PTH treatment

We hypothesized that several structural properties may predict the gain in bone mass after PTH, such as bone surface at the start of PTH treatment, bone mass at the start of PTH treatment, bone mass before ovariectomy, and amount of bone mass loss after ovariectomy. Therefore, a linear correlation was determined between several structural parameters and the gain in bone mass, gain in bone volume fraction, final bone mass, and final bone volume fraction after PTH treatment. This was done for the PTH-treated rats only.

Three-point bending of tibiae

After sacrifice, all tibiae were dissected and frozen in phosphate buffered saline solution at $-20^{\circ} \mathrm{C}$. They were thawed prior to three-point bending. The tibia was placed on the lateral surface on two rounded supporting bars with a distance of $2.4 \mathrm{~cm}$. A preload of $1 \mathrm{~N}$ was applied (ZWICK, Z020) at the medial surface of the diaphysis by lowering a third rounded bar. A constant displacement rate of $6 \mathrm{~mm} / \mathrm{min}$ was applied until failure. Displacement was measured from the actuator displacement transducer of the testing machine. From the force-displacement curve, the following mechanical parameters were determined: (1) ultimate load, defined as the maximum load, (2) displacement at ultimate load, which was corrected for the toe region, (3) extrinsic stiffness, calculated as the slope in the linear region between $40 \%$ and $80 \%$ of the ultimate load, and (4) energy to ultimate load, defined as the area under the curve until ultimate load.

\section{Statistics}

A one-way analysis of variance (ANOVA) with repeated measures was performed to compare the PTH-treated and OVX groups during treatment between weeks 8 and 14. A one-way ANOVA with a Bonferroni post hoc test was used to determine differences between the groups at certain time points, for all parameters. Furthermore, a one-way ANOVA with repeated measures was performed to compare the OVX and SHAM groups between weeks 0 and 8. Finally, an ANOVA with repeated measures was performed in the SHAM group to determine effects of aging. All $p$ values below 0.05 were considered significant.

\section{Results}

Metaphyseal structural parameters

At week 8, the ovariectomized groups displayed loss of BV/TV, Conn.D, Tb.N, and Tb.Th and an increase in SMI and Tb.Sp, indicating the development of osteopenia (Fig. 2). Beyond 8 weeks, the untreated OVX group showed further deterioration of bone structure except for Tb.Th, which increased.

Daily injections with PTH reversed the effects of OVX and prevented further deterioration of bone structure. PTH-treated animals displayed a crude plate-like trabecular bone structure and bone marrow cavity was reduced compared to OVX rats. Over the course of weeks 8 to 14 , a significant effect of time, effect of PTH treatment, and an interaction of PTH treatment and time were found for all structural parameters. PTH directly led to an increase in $\mathrm{BV} / \mathrm{TV}$ accompanied by an increase in $\mathrm{Tb}$.Th and prevention of further loss of $\mathrm{Tb} . \mathrm{N}$ and further increase of Tb.Sp. This increase in BV/TV and Tb.Th was linear and continued until sacrifice. Loss of Conn.D was prevented and SMI decreased by PTH treatment. In the time frame of weeks 8 to 10 , an interaction of PTH treatment and time was found, indicating that the effects of PTH were present within 2 weeks. After 2 weeks of PTH treatment, all structural parameters were already significantly different from the OVX group. After 6 weeks of PTH treatment, BV/TV and SMI were not significantly different between the PTH and SHAM groups. Tb.N and Conn.D were significantly lower and $\mathrm{Tb}$.Th and $\mathrm{Tb}$.Sp were significantly higher in the PTH than in the SHAM group. In the SHAM group, BV/TV, Conn.D, and Tb.N were significantly decreased and $\mathrm{Tb} . \mathrm{Sp}$ significantly increased over time as a result of aging.

Epiphyseal structural parameters

At week 8, the ovariectomized groups displayed loss of BV/TV, Conn.D, and Tb.N and an increase in SMI and Tb. $\mathrm{Sp}$, indicating the development of osteopenia (Fig. 3). Changes in the epiphysis, however, were much smaller than in the metaphysis. Beyond 8 weeks, the untreated OVX group showed further deterioration of bone structure except for $\mathrm{Tb}$.Th, which gradually increased over time. 

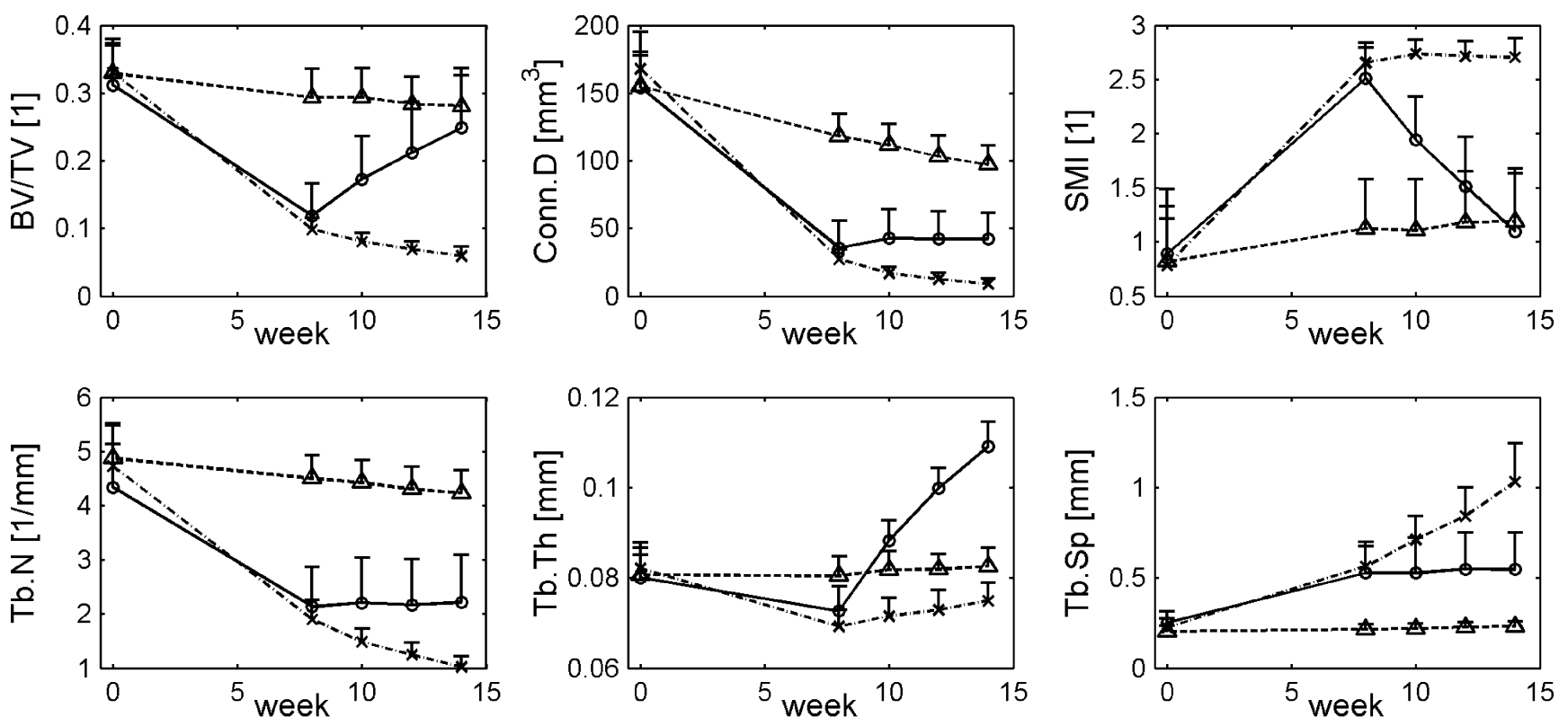

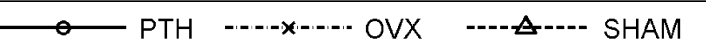

Fig. 2 Structural parameters in the metaphyseal proximal tibia for all groups at all time points (mean \pm standard deviation)

Over the course of weeks 8 to 14, a significant interaction of PTH treatment and time was found for all structural parameters except for Conn.D. PTH treatment led to a direct increase in bone volume fraction, accompanied by increases in Tb.N and Tb.Th, while Tb.Sp decreased. This increase in $\mathrm{BV} / \mathrm{TV}$ and Tb.N was linear and continued until sacrifice, while the increase in Tb.Th waned over time. SMI decreased after PTH treatment, while loss of Conn.D was not prevented. In the time frame of weeks 8 to 10 , a significant interaction of PTH treatment and time was found for all structural parameters except for Conn.D, indicating that the effects of PTH were present within 2 weeks. After 2 weeks of PTH treatment, BV/TV and SMI were already significantly different from the OVX group and not significantly different from the SHAM group while Tb.Th was significantly higher in the PTH group than the OVX and SHAM groups. After
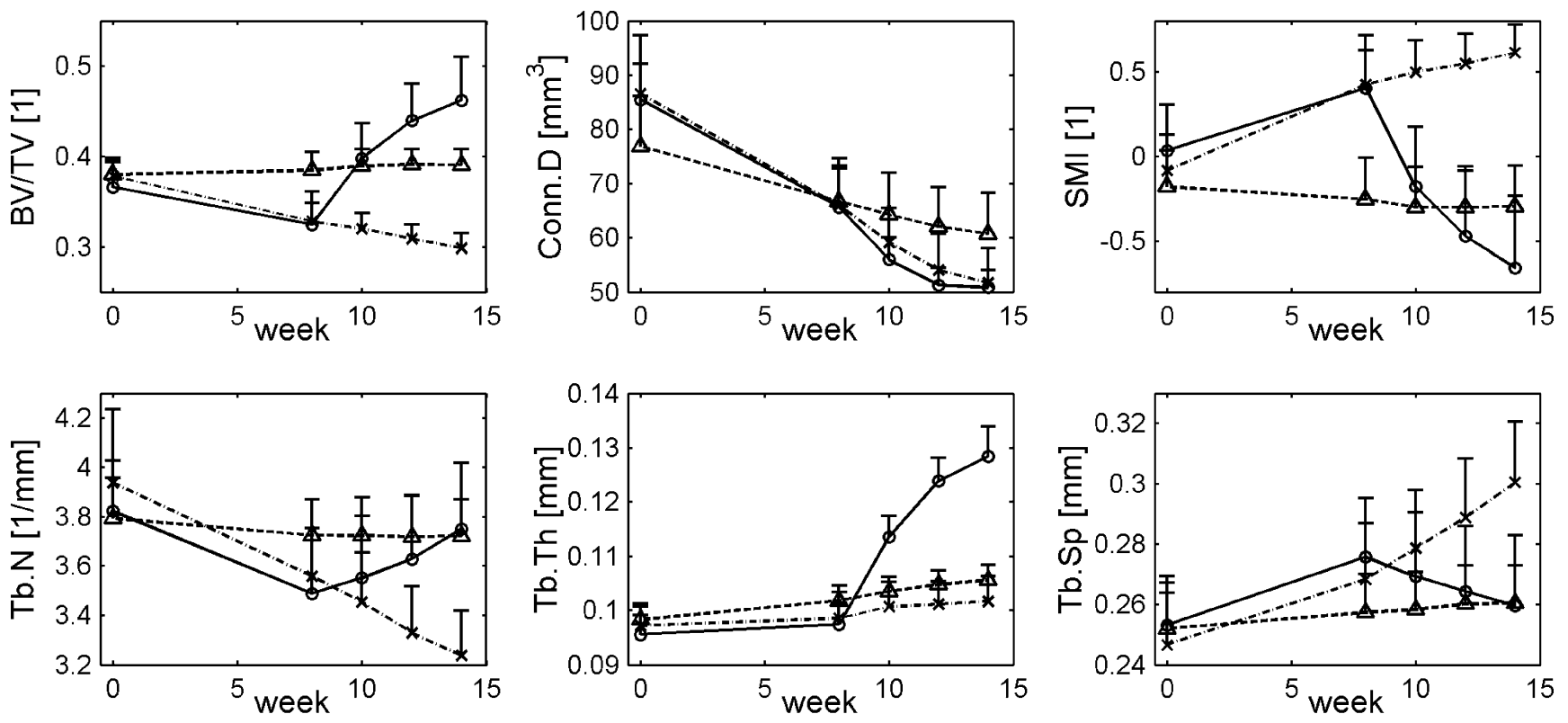

$\longrightarrow$ PTH

Fig. 3 Structural parameters in the epiphyseal, proximal tibia for all groups at all time points (mean \pm standard deviation) 
6 weeks of PTH treatment, BV/TV and Tb.Th were significantly higher than the SHAM group and than baseline values. SMI, Tb.N, and Tb.Sp were the same as the SHAM group, while Conn.D remained reduced. In the SHAM group, Conn.D significantly decreased and Tb.Th significantly increased over time as a result of aging.

Cortical thickness and polar moment of inertia in the metaphysis and diaphysis

Cortical thickness and the polar moment of inertia in the metaphysis did not significantly change within the 8 weeks after OVX compared to the SHAM group (Fig. 4). PTH treatment led to a sharp linear increase in cortical thickness and pMOI, which were both significantly different from the OVX group over time. Visual inspection of registered images of weeks 8 and 14 showed that bone formation was slightly more due to endosteal than periosteal apposition and that bone formation did not take place on all parts of the surface in the same degree (Fig. 5).

Cortical thickness in the diaphysis increased after OVX almost reaching significance $(p=0.07)$. PTH treatment led to an even sharper increase, which was linear over time and significantly different from the untreated group. The pMOI increased significantly after OVX in the first 8 weeks. After
8 weeks, this increase waned in the OVX group, while it increased significantly more in the PTH-treated group. Visual inspection of registered images of weeks 8 and 14 showed that bone formation was slightly more due to periosteal than endosteal apposition and that bone formation had taken place quite evenly over the whole surface. Cortical thickness and pMOI significantly and gradually increased over time in the metaphysis and the diaphysis of the SHAM group as a result of aging.

Mineralization of meta- and epiphyseal trabecular bone tissue and meta- and diaphyseal cortical bone tissue

At the start of the experiment, CT-estimated bone mineral density in the metaphyseal trabecular and cortical bone tissue was significantly higher in the SHAM group than in the other groups. However, because of the use of follow-up data and repeated measures design, we were still able to determine significant effects of OVX and PTH on bone mineral density. Compared to SHAM, OVX was found to lead to a significantly lower increase in mineral density of meta- and diaphyseal, cortical bone tissue over the first 8 weeks, but did not significantly affect trabecular bone tissue (Fig. 6). Over weeks 8 to 14 , the meta- and epiphyseal trabecular bone tissue of the PTH group was found to have a significantly
Fig. 4 Cortical thickness and polar moment of inertia ( $p M O I)$ in the meta- and diaphysis of the tibia for all groups at all time points (mean \pm standard deviation)
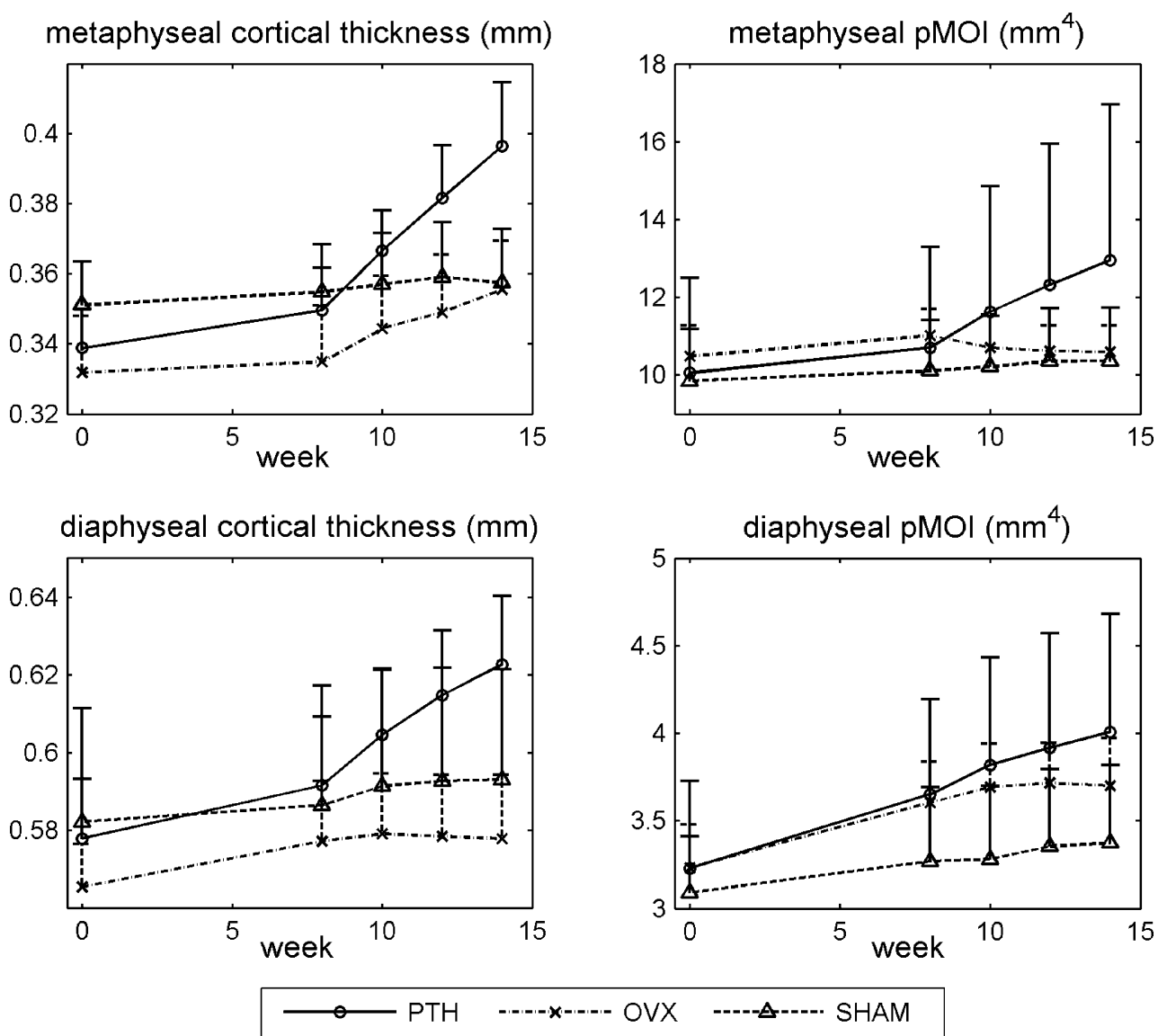

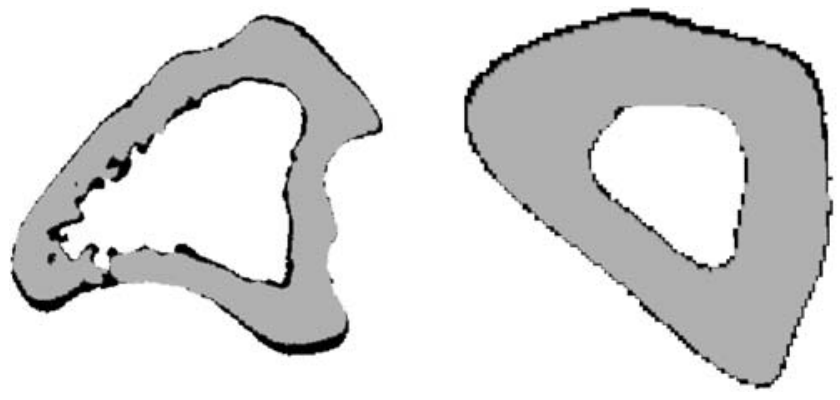

Fig. 5 Registered images of metaphyseal (left) and diaphyseal (right) cortical bone taken at weeks 8 and 14 showing bone formation during 6 weeks in the cortex of a PTH-treated rat. Gray is bone at week 8, black is newly formed bone

more increasing bone mineral density than that of the OVX group. Cortical bone mineral density was not affected by PTH treatment. Bone mineral density of all measured bone areas was found to significantly increase over time in the SHAM group.

Trabecular tunneling and remodeling

For all PTH-treated rats, no cases of trabecular tunneling were detected in metaphyseal trabecular bone between weeks 12 and 14. For several rats, one trabecula was selected and analyzed as it developed over time after PTH treatment. Figure 7 shows how PTH in this particular trabecula first led to filling and overfilling of cavities, while later, more bone was added to the surface of the trabecula resulting in a thicker trabecula. Also, resorption still appeared to take place in this trabecula. Another trabecula after segmentation of the image appeared cleaved due to OVX-induced increased resorption. PTH treatment led to bone formation, which took place where it was most beneficial, i.e., at the cleaved site, restoring the trabecula. This indicates that there probably was still a thin line of bone left in the center, which was unaccounted for after segmentation, but large enough for bone formation to take place. It was found that for all rats, the maximum trabecular thickness continued to increase over time. Therefore, no maximum limit for trabecular thickness appeared to be present.

Prediction of gain in bone mass after PTH treatment

The linear correlations between several structural parameters and the gains in bone mass, gain in bone volume fraction, final bone mass, and final bone volume fraction after PTH treatment varied between the specific parameters as well as bone regions (Table 1). More significant
Fig. 6 CT-estimated bone mineral density of metaphyseal trabecular bone, metaphyseal cortical bone, diaphyseal cortical bone, and epiphyseal trabecular bone (mean \pm standard deviation)
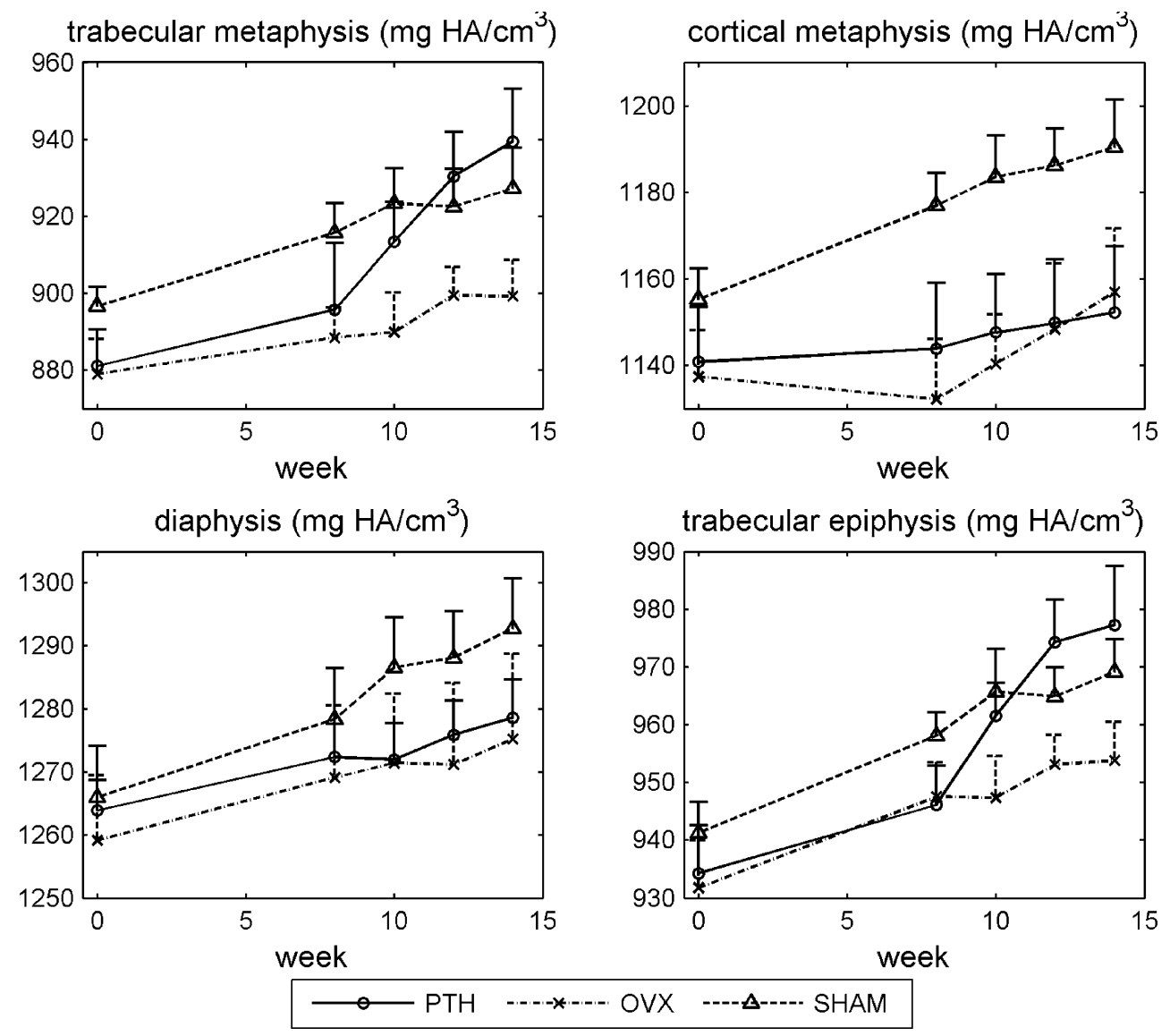

cortical metaphysis $\left(\mathrm{mg} \mathrm{HA} / \mathrm{cm}^{3}\right.$ )

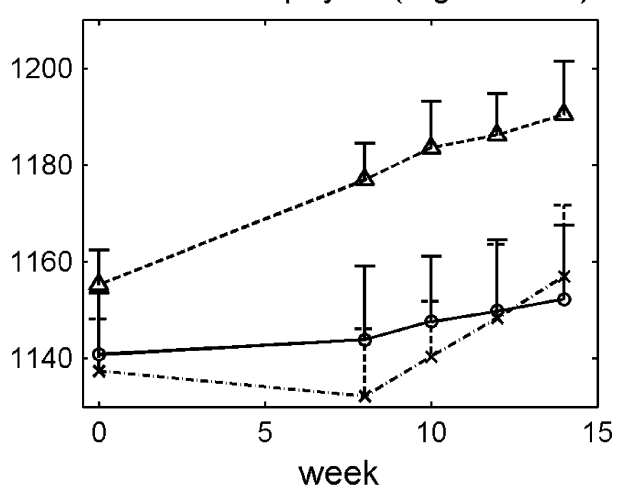



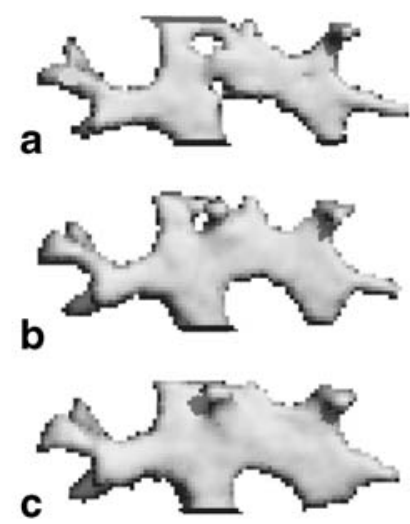

e
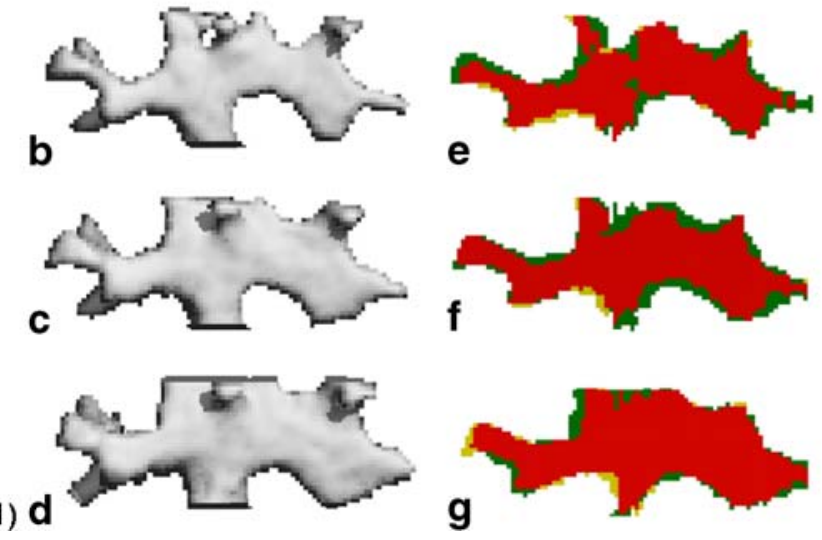

Fig. 7 A trabecula in two PTH-treated ovariectomized rats was tracked over time to determine the development of bone formation ( 1 and 2). On the left of 1 and 2, you see three-dimensional segmented images of a trabecula, after PTH treatment is started at week 8 , taken at weeks $8(a)$, $10(b), 12(c)$, and $14(d)$. On the right, you see overlaid twodimensional segmented sections comparing weeks 8 and $10(e), 10$ and $12(f)$, and 12 and $14(\mathrm{~g})$. Yellow indicates resorbed bone, green newly

predictions were found for the metaphysis than the epiphysis. Best correlations were found between BV and $\mathrm{BV} / \mathrm{TV}$ at week 0 and $\mathrm{BV}$ and $\mathrm{BV} / \mathrm{TV}$ at week 14, respectively, in both the meta- and epiphysis. Paradoxically, the loss of bone after OVX did not predict the gain of bone after PTH treatment well. From structural parameters evaluated at week 8 , bone surface (BS) was the best predictor of the gain in bone after PTH. While BV and BV/ TV at week 8 predicted the gain in bone mass over the next 6 weeks quite well, the predictive value of $\mathrm{BV}$ and $\mathrm{BV} / \mathrm{TV}$
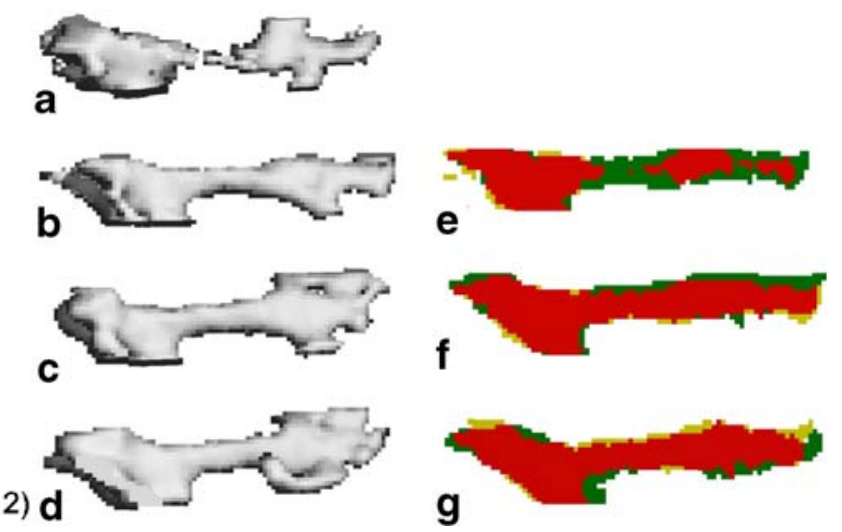

formed bone, and red unchanged bone. Bone formation is clearly seen over time in both trabeculae. In trabecula 1 , bone is mostly deposited in the cavities in the first 2 weeks, while later on bone is added to the surface. In trabecula 2, the trabecula appears cleaved after segmentation, although most likely there was still a thin line of bone present. PTH treatment leads to bone formation at the cleaved site, where it is most needed hereby restoring the trabecula

at weeks 8,10 , and 12 for the following 2 weeks was poor to nonsignificant. Interestingly, in the epiphysis, the slopes of these relations were negative, indicating that the higher $\mathrm{BV}$ and $\mathrm{BV} / \mathrm{TV}$, the lower the gain. All other significant relations had a positive slope.

Three-point bending of tibiae

Ultimate load and energy in the PTH group were significantly higher than in the SHAM group (Fig. 8).

Table 1 Linear correlation between several structural parameters to predict gain in bone mass, gain in bone volume fraction, final bone mass, or final bone volume fraction

\begin{tabular}{|c|c|c|c|c|c|}
\hline \multirow[t]{2}{*}{ Predictive variable } & \multirow[t]{2}{*}{ Outcome variable } & \multicolumn{2}{|c|}{ Metaphysis } & \multicolumn{2}{|c|}{ Epiphysis } \\
\hline & & $r^{2}$ & Slope & $r^{2}$ & Slope \\
\hline BS at weeks 8,10 , and 12 & $\Delta \mathrm{BV} / \mathrm{TV}$ over weeks $8-10,10-12$, and $12-14$ & 0.42 & 0.0003 & 0.23 & 0.0011 \\
\hline BS at weeks 8,10 , and 12 & $\Delta \mathrm{BV}$ over weeks $8-10,10-12$, and $12-14$ & 0.40 & 0.0077 & n.s. & - \\
\hline $\mathrm{BV} / \mathrm{TV}$ at weeks 8,10 , and 12 & $\Delta \mathrm{BV} / \mathrm{TV}$ over weeks $8-10,10-12$, and $12-14$ & n.s. & - & 0.41 & -0.23 \\
\hline BV at weeks 8,10 , and 12 & $\Delta \mathrm{BV}$ over weeks $8-10,10-12$, and $12-14$ & 0.21 & 0.13 & 0.25 & -0.21 \\
\hline$\Delta \mathrm{BV} / \mathrm{TV}$ over weeks $\quad 0-8$ & $\Delta \mathrm{BV} / \mathrm{TV}$ over weeks $8-14$ & n.s. & - & n.s. & - \\
\hline$\Delta \mathrm{BV}$ over weeks $0-8$ & $\Delta \mathrm{BV}$ over weeks $8-14$ & 0.48 & 0.95 & n.s. & - \\
\hline BS at week 8 & $\Delta \mathrm{BV} / \mathrm{TV}$ over weeks $8-14$ & 0.86 & 0.0012 & n.s. & - \\
\hline BS at week 8 & $\Delta \mathrm{BV}$ over weeks $8-14$ & 0.77 & 0.030 & n.s & - \\
\hline $\mathrm{BV} / \mathrm{TV}$ at week 8 & $\Delta \mathrm{BV} / \mathrm{TV}$ over weeks $8-14$ & 0.66 & 0.76 & n.s. & - \\
\hline BV at week 8 & $\Delta \mathrm{BV}$ over weeks $8-14$ & 0.69 & 0.88 & n.s. & - \\
\hline $\mathrm{BV} / \mathrm{TV}$ at week 0 & $\mathrm{BV} / \mathrm{TV}$ at week 14 & 0.81 & 1.3 & 0.85 & 1.6 \\
\hline BV at week 0 & BV at week 14 & 0.89 & 1.3 & 0.93 & 0.96 \\
\hline
\end{tabular}


Fig. 8 Ultimate load, ultimate displacement, extrinsic stiffness, and energy determined from three-point bending test on tibiae after sacrifice at 14 weeks. ${ }^{*} p<0.05$ compared to SHAM

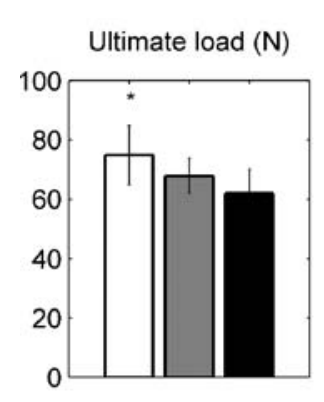

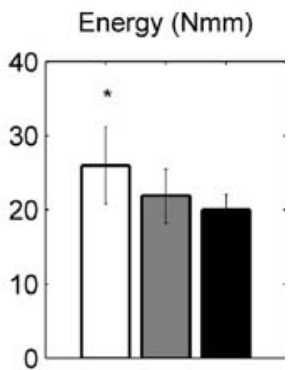

Ultimate load and energy in the OVX group tended to be slightly higher and lower than the SHAM and PTH group, respectively, though this did not reach significance. No significant differences were found in extrinsic stiffness and ultimate displacement between all groups, although the trend between groups in extrinsic stiffness was similar to the trend in ultimate load.

\section{Discussion}

For the first time, the effects of PTH treatment on trabecular and cortical bone were analyzed longitudinally with an in vivo micro-CT scanner in the same ovariectomized rats for 6 weeks. It was found that (1) PTH treatment led to constant linear increases in trabecular bone mass of the meta- and epiphysis due to linearly increasing trabecular thickness in both regions and linearly increasing trabecular number in the epiphysis, (2) a different response to PTH was seen between the meta- and epiphysis in terms of structural parameters, (3) PTH resulted in increased trabecular tissue mineralization and unaltered cortical tissue mineralization as well as slightly improved bending properties, (4) for those trabeculae analyzed, bone was formed initially on places where it was most beneficial for increasing their strength and later on to all surfaces, and (5) bone mass after PTH treatment for each rat individually was best predicted by bone mass at the start of the experiment.

In the metaphyseal trabecular bone, PTH treatment led to a constant linear increase in bone volume fraction during 6 weeks accompanied by a constantly increasing trabecular thickness and an inhibition of further loss of trabecular number. Although this is the first in vivo report on bone structural parameters, our results agree with previous crosssectional studies on the eventual effects of PTH on trabecular metaphyseal bone [8, 10-15, 22] and with an in vivo report on changes in bone mineral density [37]. In the epiphyseal trabecular bone, PTH treatment also led to an increasing bone volume fraction, accompanied by a linearly increasing trabecular number while trabecular thickness also increased, which waned over time. Previously, preventive treatment with PTH (at time point of OVX) in ovariectomized rats led to an increased bone volume fraction, trabecular number, and thickness in the tibial epiphysis, compared to untreated OVX and SHAM rats in a crosssectional study [38], though exact values were not reported. This concurs, however, with the increases that we found after recovering treatment (after osteopenia) with PTH in the epiphysis. For the first time now, bone microstructure in the epiphysis over time was reported after PTH use. The increase in bone volume fraction after PTH treatment over 6 weeks in the meta- and epiphysis was almost exactly the same. This increase resulted in the epiphysis in values that were above SHAM level while in the metaphysis values were still below SHAM. This similar increase suggests that the anabolic response to PTH is comparable in both locations.

Interestingly, the response to PTH treatment was slightly different between the meta- and epiphyseal trabecular bone, with the most striking difference being an increasing trabecular number in the epiphysis, while it stayed constant in the metaphysis. There are several possible explanations for this difference between the meta- and epiphysis and for the increase in trabecular number in the epiphysis. The deterioration of bone mass and structure after ovariectomy in the epiphysis was much smaller than in the metaphysis. Therefore, at the start of PTH treatment, the state of the bone was quite different between the meta- and epiphysis, with the latter one having a higher trabecular thickness and structure model index. It has been suggested that after PTH treatment, trabeculae will initially become thicker until a certain maximum thickness is reached [23]. Trabecular tunneling would then take place, after which thick trabeculae are cleaved into two smaller ones, which has been shown to occur in different species [19, 20, 23-25]. This implies that trabeculae will never grow beyond a certain maximum thickness, the value of which may depend on species and anatomical site. As trabecular thickness was higher in the epi- than the metaphysis, this could be an explanation for the increase in trabecular number in the epiphysis and the absence of this in the metaphysis. To test this, we analyzed the distribution of trabecular thickness in the epiphysis of 
all rats during PTH treatment. It was found that the maximum trabecular thickness continued to increase until week 14. This therefore does not support the idea of a maximum intrinsic trabecular thickness. This is further supported by the fact that trabecular thickness in the metaphysis at the final time point was higher than in the epiphysis, while trabecular number did not increase. Also, no cases of tunneling were seen in the epiphysis after visual inspection. Another explanation could lie in the decrease of total volume of interest over time in the epiphysis seen in the CT scans due to endosteal apposition. In theory, it could be that the number of trabeculae in the area close to the cortex is lower than average. This would suggest that merely a decrease in total volume would lead to an increase in trabecular number. We analyzed this possibility by using the hand-drawn contour file from week 14 for the CT scan of week 8, which excludes the outer trabecular region. We then analyzed bone structural parameters again and found that trabecular number was not increased compared to when using the original contour file for week 8 , and therefore, this possibility is excluded. Another option is that the relatively large amount of the plate-like bone enables trabecular tunneling in a different fashion than previously reported in rod-like bone by fenestration of plates, which may be difficult to see in the CT scans. A final possibility is that after 8 weeks, thin trabeculae were removed during segmentation. When these trabeculae increased in thickness, they were included resulting in an increased trabecular number at 14 weeks. This phenomenon is shown in Fig. 7.

Tissue mineral density of meta- and epiphyseal trabecular bone significantly increased over time after PTH treatment, while cortical bone in the meta- and diaphysis was unaffected. It has previously been found that ash density of the vertebral body, including cortical and trabecular bone, was significantly increased in PTHtreated ovariectomized rats compared to untreated ovariectomized rats already after 5 weeks, while after 16 weeks of PTH treatment, still no effects were found on the femoral, diaphyseal, and cortical bone [2]. In another study, using quantitative backscattered electron imaging to calculate the degree and homogeneity of mineralization, however, no significant effect of 5.5 months of PTH treatment was found on the cortical and trabecular bone of PTH-treated ovariectomized rats [33]. In yet another study on the longterm effects of PTH on mineralization in rats, no significant influences were found, although there was a slightly wider variation in mineralization in the bone reflecting the newly formed bone [18]. These differences in results in rats may be due to different methods applied to determine mineralization. Also, the different study durations are likely to play a role, as new bone formed in response to PTH is probably undermineralized; however, mineralization may increase thereafter. It should be noted that CT-based measurements of the degree of mineralization may be less reliable than other methods such as back-scattered electron imaging and microradiographic techniques. The unaffected cortical mineral density is supported by the bending results. Our bending data agree with three-point bending tests in the femur where an increase in ultimate load and extrinsic stiffness after PTH treatment was found in ovariectomized rats $[39,40]$. It can be seen that the trends between groups in ultimate load, extrinsic stiffness, and calculated polar moment of inertia are similar, which indicates that the polar moment of inertia was a good predictor of ultimate load and extrinsic stiffness. Ultimate displacement did not differ between all groups, which suggests that the newly formed bone was of similar quality as the old bone and indicates that PTH treatment did not lead to more brittle or ductile mechanical behavior. This is further supported by unaltered tissue mineralization values in the diaphyseal tissue, i.e., cortical bone.

Individual trabeculae were tracked over time during PTH treatment in all rats by using image registration software. With this method, we were able to monitor bone formation after PTH treatment on a microlevel and gather insight into how and where PTH treatment leads to new bone. In many trabeculae, it appeared that in the first 2 weeks, mostly cavities were filled, while later on bone was added to the outer surface. It has been suggested that increases in bone mass after PTH occur by remodeling- and modeling-based bone formation [41] and plasma markers in PTH-treated patients have shown that modeling increases directly after the onset of treatment [42]. Our data suggest that in rats, initially remodeling-based bone formation takes place, as cavities are filled with bone, while later, modeling-based bone formation is more pronounced as bone is added to the outer surface, which does not appear to have been resorbed first. This will need to be further validated. For several other trabeculae, it was seen that ovariectomy led to severe disruption of the trabecula to the point of almost complete cleavage after segmentation of the images. PTH treatment led to bone deposition there where most beneficial, resulting in full restoral of the trabecula. This could be explained by Frost's mechanostat, which states that bone is deposited where strains and stresses are the highest. Since in an almost cleaved trabecula merely a thin line of bone was present at certain locations, strains and stresses would be the highest at these locations leading to bone formation there. This suggests that PTH-induced bone formation is, at least in part, mechanically driven. This agrees with results from two finite element studies on CT scans of osteoporotic patients treated with teriparatide (rhPTH (1-34)), in which it was found that bone was mostly deposited on locations of high strains [30] and that teriparatide increased vertebral 
strength by altering the distribution of density within the vertebra [43].

It is clinically relevant to be able to predict to what extent a patient will respond to PTH in order to determine the best treatment. In a clinical study, several characteristics like BMD before treatment and age were examined for correlations with the increase in BMD after PTH treatment; however, no strong correlations were found [44]. In our study, the best predictor of final bone mass and bone volume fraction in both the meta- and epiphysis was bone mass and bone volume fraction at the start of the experiment, before ovariectomy. If these results would be translational to clinical practice, which needs to be tested, this would indicate that bone mineral density before menopause would predict bone mineral density after PTH treatment of osteoporotic patients.

Cortical bone mass increased linearly over time after PTH treatment in the meta- and diaphysis while marrow cavity volume decreased. In several cross-sectional studies, in which the effect of between 8 weeks and 6 months of PTH treatment was evaluated in ovariectomized rats, an increase in cortical bone mass was found $[6,14,38]$. In a study in ovariectomized mice, it was found that within 3 weeks of PTH treatment, cortical thickness was significantly increased in the metaphysis and after 7 weeks, cortical thickness was even higher [45]. Diaphyseal cortical thickness was significantly increased only after 7 weeks of treatment. In another study, the effects of PTH treatment on metaphyseal cortical thickness of the tibia in ovariectomized rats was studied over time by using peripheral quantitative computed tomography (pQCT) [46]. A linear increase in cortical thickness was found until about 6 weeks, after which the effect reached a plateau. Taken together, our linear increase in dia- and metaphyseal cortical bone after PTH treatment agrees with the literature. In the metaphysis, no effect of ovariectomy was found on cortical bone parameters, which agrees with previous studies [47, 48]. Interestingly, cortical thickness and polar moment of inertia in the diaphysis increased after ovariectomy, which is in contrast to significant decreases $[21,49]$ and no significant changes $[50,51]$ previously reported.

It has previously been found that PTH leads to a predominance of endocortical over periosteal bone apposition in cortical bone [16-18, 52]. Based on registered images of weeks 8 and 14, before and after PTH treatment, we found that endosteal and periosteal bone apposition both took place in the meta- and diaphysis, with a slight predominance of endosteal formation in the former one and a slight predominance of periosteal formation in the latter one. This difference between the meta- and diaphysis could be related to the following. It is seen after ovariectomy that periosteal bone apposition increases and bone marrow cavity increases due to endocortical resorption.
PTH treatment would add to this periosteal expansion resulting in a relatively higher periosteal bone formation rate compared to the metaphysis. It is also possible that the increased endocortical metaphyseal bone is the result of "corticalization" of the subcortical trabecular elements. We also saw that while the degree of bone apposition was evenly distributed over the endo- and periosteal surface of the diaphysis, it varied quite largely over the endo- and periosteal surface of the metaphysis. This could indicate that bone apposition is stimulated more in certain locations than others, which may also partly be the result of remodeling due to linear growth, which still is present in the adult rat $[28,53]$.

This study was limited by a treatment period with PTH of 6 weeks. It was found that bone volume fraction in the meta- and epiphyseal trabecular bone and cortical thickness in the meta- and diaphysis continued to increase linearly. It is very likely though that these increases will wane after a longer treatment period. Although no trabecular tunneling was detected, it would be interesting to determine how trabecular structure would develop further over time as bone mass continues to increase. Another limitation lies in the translation of our rat study to clinical practice. It is known that rat cortical bone is not subject to Haversian remodeling [28], which has shown to lead to different responses to PTH compared to species with Haversian remodeling, in which negative $[54,55]$ and no effects $[56$, 57] on cortical thickness were found. Also, rats in our study were subjected to serial radiation resulting from CT scanning; however, we have previously shown that eight weekly scans do not lead to detectable radiation damage [36]. Since the total number of scans in this study was six and the shortest interval between scans was 2 weeks, we do not expect any radiation damage. Finally, concern has been raised regarding the predictive value of CT-derived tissue mineralization $[58,59]$. It could be that thicker trabeculae would lead to more beam hardening effects, which would result in a lower average mineralization. The fact that we found an increased mineralization degree indicates that this is most likely not due to beam hardening. An explanation for our results could be that when trabeculae thicken after PTH treatment, the center is not being remodeled anymore resulting in an increased mineralization of this bone. The algorithm calculating the mineralization peels off two voxels of the outside of the bone, which is probably the new less mineralized bone. This is thus not incorporated in the calculation, which could result in the increased mineralization.

This study demonstrated that 6 weeks of PTH treatment of osteopenic rats led to increases in bone volume fraction of the tibial meta- and epiphysis as well as increases in cortical bone mass of the tibial meta- and diaphysis. By using an in vivo micro-CT method, it was shown that net bone 
formation started directly after the onset of treatment and continued with the same rate for at least 6 weeks in both trabecular and cortical bone. Deposition of bone appeared to be mechanically driven, resulting in cleaved trabeculae being fully restored again. The increase in bone volume fraction was similar in the meta- and epiphysis; however, the resulting changes in microstructure were different, which may have different mechanical implications.

Acknowledgments This work was funded by The Netherlands Organisation for Scientific Research (NWO). We thank Jo Habets and Leonie Niesen for performing the ovariectomies, giving daily PTH injections and the animal care. We thank Rianne Reinartz and Anthal Smits for contouring.

Conflicts of interest Dr. van Rietbergen serves as a consultant for Scanco Medical AG. All other authors state that they have no conflicts of interest.

Open Access This article is distributed under the terms of the Creative Commons Attribution Noncommercial License which permits any noncommercial use, distribution, and reproduction in any medium, provided the original author(s) and source are credited.

\section{References}

1. Mosekilde L, Thomsen JS, McOsker JE (1997) No loss of biomechanical effects after withdrawal of short-term PTH treatment in an aged, osteopenic, ovariectomized rat model. Bone 20:429-437

2. Sogaard CH, Mosekilde L, Thomsen JS, Richards A, McOsker JE (1997) A comparison of the effects of two anabolic agents (fluoride and PTH) on ash density and bone strength assessed in an osteopenic rat model. Bone 20:439-449

3. Li M, Mosekilde L, Sogaard CH, Thomsen JS, Wronski TJ (1995) Parathyroid hormone monotherapy and cotherapy with antiresorptive agents restore vertebral bone mass and strength in aged ovariectomized rats. Bone 16:629-635

4. Mosekilde L, Danielsen CC, Sogaard CH, McOsker JE, Wronski TJ (1995) The anabolic effects of parathyroid hormone on cortical bone mass, dimensions and strength - assessed in a sexually mature, ovariectomized rat model. Bone 16:223-230

5. Mosekilde L, Danielsen CC, Sogaard CH, Thorling E (1994) The effect of long-term exercise on vertebral and femoral bone mass, dimensions, and strength — assessed in a rat model. Bone 15:293301

6. Baumann BD, Wronski TJ (1995) Response of cortical bone to antiresorptive agents and parathyroid hormone in aged ovariectomized rats. Bone 16:247-253

7. Wronski TJ, Yen C-F (1994) Anabolic effects of parathyroid hormone on cortical bone in ovariectomized rats. Bone 15:51-58

8. Meng XW, Liang XG, Birchman R, Wu DD, Dempster DW, Lindsay R, Shen V (1996) Temporal expression of the anabolic action of PTH in cancellous bone of ovariectomized rats. J Bone Miner Res 11:421-429

9. Oxlund H, Andreassen TT (2004) Simvastatin treatment partially prevents ovariectomy-induced bone loss while increasing cortical bone formation. Bone 34:609-618

10. Kasukawa Y, Miyakoshi N, Itoi E, Tsuchida T, Tamura Y, Kudo T, Suzuki K, Seki A, Sato K (2004) Effects of h-PTH on cancellous bone mass, connectivity, and bone strength in ovariectomized rats with and without sciatic-neurectomy. J Orthop Res 22:457-464

11. Zhang KQ, Chen JW, Li QN, Li GF, Tian XY, Huang LF, Bao LH, Wang ML (2002) Effect of intermittent injection of recombinant human parathyroid hormone on bone histomorphometry of ovariectomized rats. Acta Pharmacol Sin 23:659-662

12. Lane NE, Yao W, Kinney JH, Modin G, Balooch M, Wronski TJ (2003) Both hPTH(1-34) and bFGF increase trabecular bone mass in osteopenic rats but they have different effects on trabecular bone architecture. J Bone Miner Res 18:2105-2115

13. Nozaka K, Miyakoshi N, Kasukawa Y, Maekawa S, Noguchi H, Shimada Y (2008) Intermittent administration of human parathyroid hormone enhances bone formation and union at the site of cancellous bone osteotomy in normal and ovariectomized rats. Bone 42:90-97

14. Iwaniec UT, Moore K, Rivera MF, Myers SE, Vanegas SM, Wronski TJ (2007) A comparative study of the bone-restorative efficacy of anabolic agents in aged ovariectomized rats. Osteoporos Int 18:351-362

15. Fox J, Miller MA, Newman MK, Metcalfe AF, Turner $\mathrm{CH}$, Recker RR, Smith SY (2007) Daily treatment of aged ovariectomized rats with human parathyroid hormone (1-84) for 12 months reverses bone loss and enhances trabecular and cortical bone strength. Bone 41:321-330

16. Ejersted C, Andreassen TT, Hauge E-M, Melsen F, Oxlund H (1995) Parathyroid hormone (1-34) increases vertebral bone mass, compressive strength, and quality in old rats. Bone 17:507-511

17. Gasser JA (1997) Quantitative assessment of bone mass and geometry by pQCT in rats in vivo and site specificity of changes at different skeletal sites. J Jpn Soc Bone Morphometry 7:107-114

18. Kneissel M, Boyde A, Gasser JA (2001) Bone tissue and its mineralization in aged estrogen-depleted rats after long-term intermittent treatment with parathyroid hormone $(\mathrm{PTH})$ analog SDZ PTS 893 or human PTH(1-34). Bone 28:237-250

19. Fox J, Miller MA, Newman MK, Turner CH, Recker RR, Smith SY (2007) Treatment of skeletally mature ovariectomized rhesus monkeys with PTH(1-84) for 16 months increases bone formation and density and improves trabecular architecture and biomechanical properties at the lumbar spine. J Bone Miner Res 22:260-273

20. Jerome CP, Burr DB, Van Bibber T, Hock JM, Brommage R (2001) Treatment with human parathyroid hormone (1-34) for 18 months increases cancellous bone volume and improves trabecular architecture in ovariectomized cynomolgus monkeys (Macaca fascicularis). Bone 28:150-159

21. Sato M, Ma YL, Hock JM, Westmore MS, Vahle J, Villanueva A, Turner CH (2002) Skeletal efficacy with parathyroid hormone in rats was not entirely beneficial with long-term treatment. J Pharmacol Exp Ther 302:304-313

22. Oxlund H, Dalstra M, Ejersted C, Andreassen TT (2002) Parathyroid hormone induces formation of new cancellous bone with substantial mechanical strength at a site where it had disappeared in old rats. Eur J Endocrinol 146:431-438

23. Iida-Klein A, Lu SS, Cosman F, Lindsay R, Dempster DW (2007) Effects of cyclic vs. daily treatment with human parathyroid hormone (1-34) on murine bone structure and cellular activity. Bone 40:391-398

24. Boyce RW, Paddock CL, Franks AF, Jankowsky ML, Eriksen EF (1996) Effects of intermittent hPTH (1-34) alone and in combination with1,25(OH)2D3 or risedronate on endosteal bone remodeling in canine cancellous and cortical bone. J Bone Miner Res 11:600-611

25. Miller MA, Bare SP, Recker RR, Smith SY, Fox J (2008) Intratrabecular tunneling increases trabecular number throughout the skeleton of ovariectomized rhesus monkeys treated with parathyroid hormone 1-84. Bone 42:1175-1183 
26. Guo XE, Kim CH (2002) Mechanical consequence of trabecular bone loss and its treatment: a three-dimensional model simulation. Bone 30:404-411

27. Turner CH (2002) Biomechanics of bone: determinants of skeletal fragility and bone quality. Osteoporos Int 13:97-104

28. Jee WS, Yao W (2001) Overview: animal models of osteopenia and osteoporosis. J Musculoskelet Neuronal Interact 1:193-207

29. Westerlind KC, Wronski TJ, Ritman EL, Luo ZP, An KN, Bell NH, Turner RT (1997) Estrogen regulates the rate of bone turnover but bone balance in ovariectomized rats is modulated by prevailing mechanical strain. Proc Natl Acad Sci USA 94:4199-4204

30. Graeff C, Zysset PK, Marin F, Gluer CC (2007) Bone apposition in patients on Teriparatide treatment is preferably directed to skeletal regions of local structural weakness: assessment by high resolution CT based finite element analysis in vivo. J Bone Miner Res 22:S74-S75

31. Li M, Liang H, Shen Y, Wronski TJ (1999) Parathyroid hormone stimulates cancellous bone formation at skeletal sites regardless of marrow composition in ovariectomized rats. Bone 24:95-100

32. Misof BM, Roschger P, Cosman F, Kurland ES, Tesch W, Messmer P, Dempster DW, Nieves J, Shane E, Fratzl P, Klaushofer K, Bilezikian J, Lindsay R (2003) Effects of intermittent parathyroid hormone administration on bone mineralization density in iliac crest biopsies from patients with osteoporosis: a paired study before and after treatment. J Clin Endocrinol Metab 88:1150-1156

33. Valenta A, Roschger P, Fratzl-Zelman N, Kostenuik PJ, Dunstan CR, Fratzl P, Klaushofer K (2005) Combined treatment with PTH (1-34) and OPG increases bone volume and uniformity of mineralization in aged ovariectomized rats. Bone 37:87-95

34. Mulder L, Koolstra JH, Van Eijden TM (2004) Accuracy of microCT in the quantitative determination of the degree and distribution of mineralization in developing bone. Acta Radiol 45:769-777

35. Verhulp E, van Rietbergen B, Huiskes R (2004) A three-dimensional digital image correlation technique for strain measurements in microstructures. J Biomech 37:1313-1320

36. Brouwers JEM, van Rietbergen B, Huiskes R (2007) No effects of in vivo micro-CT radiation on structural parameters and bone marrow cells in proximal tibia of wistar rats detected after eight weekly scans. J Orthop Res 25:1325-1332

37. Sato M, Vahle J, Schmidt A, Westmore M, Smith S, Rowley E, Ma LY (2002) Abnormal bone architecture and biomechanical properties with near-lifetime treatment of rats with PTH. Endocrinology 143:3230-3242

38. Sato M, Zeng GQ, Turner CH (1997) Biosynthetic human parathyroid hormone (1-34) effects on bone quality in aged ovariectomized rats. Endocrinology 138:4330-4337

39. Washimi Y, Ito M, Morishima Y, Taguma K, Ojima Y, Uzawa T, Hori M (2007) Effect of combined humanPTH(1-34) and calcitonin treatment in ovariectomized rats. Bone 41:786-793

40. Shen V, Birchman R, Wu DD, Lindsay R (2000) Skeletal effects of parathyroid hormone infusion in ovariectomized rats with or without estrogen repletion. J Bone Miner Res 15:740-746

41. Compston JE (2007) Skeletal actions of intermittent parathyroid hormone: effects on bone remodelling and structure. Bone 40:1447-1452

42. Burr DB (2005) Does early PTH treatment compromise bone strength? The balance between remodeling, porosity, bone mineral, and bone size. Curr Osteoporos Rep 3:19-24

43. Keaveny TM, Donley DW, Hoffmann PF, Mitlak BH, Glass EV, San Martin JA (2007) Effects of teriparatide and alendronate on vertebral strength as assessed by finite element modeling of
QCT scans in women with osteoporosis. J Bone Miner Res 22:149-157

44. Sellmeyer DE, Black DM, Palermo L, Greenspan S, Ensrud K, Bilezikian J, Rosen CJ (2007) Hetereogeneity in skeletal response to full-length parathyroid hormone in the treatment of osteoporosis. Osteoporos Int 18:973-979

45. Zhou H, Iida-Klein A, Lu SS, Ducayen-Knowles M, Levine LR, Dempster DW, Lindsay R (2003) Anabolic action of parathyroid hormone on cortical and cancellous bone differs between axial and appendicular skeletal sites in mice. Bone 32:513-520

46. Gasser JA (1995) Assessing bone quantity by pQCT. Bone 17: S145-S154

47. Bourrin S, Ammann P, Bonjour JP, Rizzoli R (2002) Recovery of proximal tibia bone mineral density and strength, but not cancellous bone architecture, after long-term bisphosphonate or selective estrogen receptor modulator therapy in aged rats. Bone 30:195-200

48. Shen V, Birchman R, Liang XG, Wu DD, Lindsay R, Dempster DW (1997) Prednisolone alone, or in combination with estrogen or dietary calcium deficiency or immobilization, inhibits bone formation but does not induce bone loss in mature rats. Bone 21:345-351

49. Kobayashi M, Hara K, Akiyama Y (2002) Effects of vitamin K2 (menatetrenone) on calcium balance in ovariectomized rats. Jpn $\mathrm{J}$ Pharmacol 88:55-61

50. Hara K, Kobayashi M, Akiyama Y (2007) Influence of bone osteocalcin levels on bone loss induced by ovariectomy in rats. J Bone Miner Metab 25:345-353

51. Kippo K, Hannuniemi R, Isaksson P, Lauren L, Osterman T, Peng Z, Tuukkanen J, Kuurtamo P, Vaananen HK, Sellman R (1998) Clodronate prevents osteopenia and loss of trabecular connectivity in estrogen-deficient rats. J Bone Miner Res 13:287-296

52. Mosekilde L, Tornvig L, Thomsen JS, Orhii PB, Banu MJ, Kalu DN (2000) Parathyroid hormone and growth hormone have additive or synergetic effect when used as intervention treatment in ovariectomized rats with established osteopenia. Bone 26:643651

53. Waarsing JH, Day JS, Verhaar JAN, Ederveen AGH, Weinans H (2006) Bone loss dynamics result in trabecular alignment in aging and ovariectomized rats. J Orthop Res 24:926-935

54. Slovik DM, Neer RM, Potts JTJ (1981) Short-term effects of synthetic human parathyroid hormone-(1-34) administration on bone mineral metabolism in osteoporotic patients. J Clin Invest 68:1261-1271

55. Hodsman AB, Steer BM, Fraher LJ, Drost DJ (1991) Bone densitometric and histomorphometric responses to sequential human parathyroid hormone (1-38) and salmon calcitonin in osteoporotic patients. Bone Miner 14:67-83

56. Reeve J, Davies UM, Hesp R, McNally E, Katz D (1990) Treatment of osteoporosis with human parathyroid peptide and observations on effect of sodium fluoride. Br Med J 301:314 318

57. Hodsman AB, Fraher LJ, Watson PH, Ostbye T, Stitt LW, Chi JD, Taves DH, Drost D (1997) A randomized controlled trial to compare the efficacy of cyclical parathyroid hormone versus cyclical parathyroid hormone and sequential calcitonin to improve bone mass in postmenopausal women with osteoporosis. J Clin Endocrinol Metab 82:620-628

58. Fajardo R, Cory E, Patel N, Nazarian A, Snyder B, Bouxsein ML (2007) Specimen size and porosity can introduce error into microCT-based tissue mineral density measurements. Bone 44:76-84

59. Cory E, Patel N, Nazarian A, Snyder B, Bouxsein ML, Fajardo R (2007) Effect of surrounding tissue on density evaluation via microcomputed tomography. Trans Orthop Res Soc 32:373 